\title{
Germanica
}

\section{L'empreinte de la France chez Arthur Schnitzler et Hugo von Hofmannsthal}

Traces of France in the work of Hofmannsthal and Schnitzler

Franzosisches Erbe im Schaffen von Schnitzler u. Hofmannsthal

\section{Martine Sforzin}

\section{CpenEdition}

Journals

Édition électronique

URL : http://journals.openedition.org/germanica/2119

DOI : 10.4000/germanica.2119

ISSN : 2107-0784

Éditeur

Université de Lille

Édition imprimée

Date de publication : 15 juin 2013

Pagination : $37-51$

ISBN : 9782913857315

ISSN : 0984-2632

\section{Référence électronique}

Martine Sforzin, «L'empreinte de la France chez Arthur Schnitzler et Hugo von Hofmannsthal »,

Germanica [En ligne], 52 | 2013, mis en ligne le 20 juin 2013, consulté le 06 octobre 2020. URL : http:// journals.openedition.org/germanica/2119; DOI : https://doi.org/10.4000/germanica.2119

\section{(c) Tous droits réservés}




\title{
L'empreinte de la France chez Arthur Schnitzler et Hugo von Hofmannsthal
}

\author{
Martine SFORZIN \\ Université de Valenciennes et du Hainaut-Cambrésis
}

\begin{abstract}
L'homme très élevé n'est jamais un original. Sa personnalité est aussi insignifiante qu'il le faut. Peu d'inégalités; aucune superstition de l'intellect. Pas de craintes vaines. Il n'a pas peur des analyses; il les mène - ou bien ce sont elles qui le conduisent - , aux conséquences éloignées; il retourne au réel sans effort. Il imite, il innove; il ne rejette pas l'ancien, parce qu'il est ancien; ni le nouveau, pour être nouveau; mais il consulte en lui quelque chose d'éternellement actuel.
\end{abstract}

Paul Valéry ${ }^{1}$

Il faut attendre le milieu des années quatre-vingt du siècle dernier pour assister en France à une véritable (re)découverte par le grand public de ce qu'il est convenu d'appeler la Modernité viennoise. Arthur Schnitzler et Hugo von Hofmannsthal figurent en première place pour illustrer le pendant à Vienne de la Belle Époque, quand les spécialistes élargissent l'éventail à des noms toujours aussi mal connus en France,

1. - La citation figure en exergue de l'Avant-propos pour les Écrits en prose de Hugo von Hofmannsthal par Charles Du Bos in http://www.biblisem.net/etudes/duboshof.htm. 
tels que Richard Beer-Hofmann, Leopold von Andrian, Peter Altenberg ou encore, Hermann Bahr, dont le rôle d' importateur $»^{2}$ culturel a pourtant été capital dans l'éclosion de l'avant-garde littéraire à Vienne au tournant du siècle dernier. C'est par ailleurs au travers de ses spécificités et de ses particularités que la Modernité viennoise s'est avérée refléter la quintessence des séismes qui ont alors secoué l'Europe, sonnant le glas du vieux continent et laissant à peine entrevoir sous les oripeaux de ce grand corps moribond l'ombre d'une renaissance. Aussi garderons-nous présent à l'esprit que ces deux Viennois l'étaient d'autant plus qu'ils se sentaient fils d'une même et unique matrice, l'Europe - fût-elle agonisante. Leur rêve de voir l'Europe sauvée pouvait bien, quant à lui, puiser à des sources différentes, ils le partageaient.

La bibliographie établie par Jeanne Benay en $1994^{3}$ dresse un bilan de la réception de Schnitzler en France tandis que le titre de son article « Schnitzler en France - Schnitzler et la France » laisse déjà entendre le choix de ne pas dissocier sa réception en France de la question des emprunts de Schnitzler à la France ; le choix encore de ne pas séparer la perception que cet écrivain avait lui-même de la vie littéraire et culturelle de la France de son époque, de la perception qu'il pouvait avoir de sa propre image dans ce pays. Depuis, la thèse de Wolfgang Sabler sur le théâtre de Schnitzler ${ }^{4}$, l'ouvrage de Jacques Le Rider Arthur Schnitzler ou la Belle Époque viennoise ${ }^{5}$, sont venus augmenter la bibliographie de J. Benay et l'absence d'une étude systématique et approfondie vient d'être comblée par l'ouvrage de K. Zieger, Enquête sur une réception. Arthur Schnitzler et la France 1894-19386. Vu de l'étranger, Schnitzler est légitimement associé à son contemporain Hofmannsthal mais une fois ces deux écrivains rangés dans la catégorie de représentants phares de la Jeune Vienne, on ne saurait se satisfaire de cette assimilation sans nuance. Une comparaison rapide avec Schnitzler laisse apparaître que les relations de Hofmannsthal à la France ont été étudiées depuis une plus longue date. La publication de la thèse de Francis Claudon remonte

2. - Cf. l'article de Blaise Wilfert « Cosmopolis et l'homme invisible. Les importateurs de littérature étrangère en France 1885-1914 », Le Seuil, Actes de la recherche en sciences sociales $2002 / 4, n^{\circ} 144$, p. 33 à 46 . Article disponible en ligne sur http://www.cairn.info/revue-actes-de-la-recherche-en-sciences-sociales-2002-4-page-33. htm.

3. - Cf. Austriaca n³9, décembre 1994, Arthur Schnitzler (études réunies par Jacques Le Rider, Gilbert Ravy et Sigurd Scheichl), Université de Rouen, p. 125-155.

4. - Wolfgang Sabler, Arthur Schnitzler. Écriture dramatique et conventions théâtrales, Bern, Peter Lang, Collection Contacts, Série I - Theatrica. 21, 2002.

5. - Jacques Le Rider, Arthur Schnitzler ou la Belle Époque viennoise, Paris, Belin, 2003.

6. - Cf. Karl Zieger, Enquête sur une réception. Arthur Schnitzler et la France 1894-1938, Villeneuve d'Ascq, Presses Universitaires du Septentrion, 2012. 
à $1979^{7}$ et suit la voie ouverte par Charles Du Bos qui dans son Journal et ses essais (Approximations) avait révélé Hofmannsthal au public français, l'élevant aux mêmes sommets de la littérature européenne qu'un Paul Valéry, un Paul Claudel ou encore un Marcel Proust ${ }^{8}$.

Toutefois, notre propos ici n'est pas tant d'approfondir cette question de la réception de Schnitzler ou de Hofmannsthal en France que de déterminer, à l'inverse, en quoi ces deux écrivains ont pu se nourrir d'un lien à la France rendu intime, au-delà de leurs relations d'amitié, ou d'impressions de voyages, par la connaissance que chacun avait de la littérature française. Si nous préférons parler d'empreinte, c'est moins pour repérer et saisir une quelconque influence de la France sur ces deux auteurs que pour analyser la dynamique, le mouvement d'interpénétration qui a pu jouer dans ces relations. Ainsi, empreinte ne signifie pas pour nous une marque indélébile qui éclipserait ou interdirait toute originalité, mais une trace laissée de manière plus ou moins visible, une rémanence dont les effets ou le ferment ne seraient pas nécessairement conformes à l'original.

Nous ne reviendrons pas ici sur les limites inhérentes à la notion d'influence telles qu'elles ont été mises en évidence par Michel Espagne et Michael Werner dans leurs travaux sur les transferts culturels. Nous rappellerons simplement, pour aller dans leur sens, que la plupart des drames de Schnitzler, par exemple, éclipsent tout un pan du théâtre de boulevard français parfaitement déclassé aujourd'hui ; nous verrons par ailleurs en quoi le Schnitzler prosateur peut légitimement récuser l'idée d'être un épigone. Nous garderons présent à l'esprit le mouvement d'échange dynamique qui sous-tend tout ancrage d'un écrivain dans un champ littéraire donné; nous n'oublierons pas que dans le cas précis de Schnitzler, cette dynamique s'intensifie encore par le fait qu'elle vient se réfléchir dans l'attention pointilleuse que Schnitzler portait à la diffusion et à la réception de ses œuvres.

Établir un simple parallèle entre les relations de Schnitzler à la France et celles de Hofmannsthal à ce même pays pourrait nous entraîner à retrouver chez le premier, le subtil sondeur des âmes maintes fois loué, doublé du critique clairvoyant des turpitudes de la société de son temps, chez le second, une sorte d'esthète très tôt repenti qui ferait amende honorable en appelant à une révolution conservatrice. Afin d'éviter le

7. - Francis Claudon, Hofmannsthal et la France, Berne, Éditions Peter Lang, 1979.

8. - F. Claudon va jusqu'à se demander s'il s'est trouvé en Autriche un seul critique qui ait reconnu dans ces proportions l'importance et le génie de Hofmannsthal. Cf. l'article de cet auteur « Die Rezeption Hofmannsthals durch Charles Du Bos », in Wolfram Mauser (Hrsg.), Hofmannsthal und Frankreich, Hofmannsthal-Forschungen Band 9, Freiburg i. Br., 1987, p. 201. 
risque de ces redites, voire de ces clichés, nous tenterons de mettre en abyme les liens de chacun à la France, pour faire se réfracter dans ces prismes différents l'inscription particulière, le cheminement de chacun dans la voie de la modernité. La démarche devrait, à partir de ce « horschamp », révéler une vision et une compréhension moins monolithique, moins uniformisante de la modernité de ces deux Viennois.

Dans l'espace qui nous est imparti ici nous nous en tiendrons à deux éléments de l'empreinte française à partir desquels aussi bien Hofmannsthal que Schnitzler se sont affirmés, chacun à sa manière, comme représentants de la modernité: la langue française d'une part et, liée à la question de la langue, la fonction éthique de la littérature.

Il n'est pas abusif d'avancer que le premier lien véritable, tant de Schnitzler que de Hofmannsthal à la France, se tisse à partir du goût que chacun cultive de la langue française. Cette première forme d'ouverture sur l'étranger est en effet un élément caractéristique de la bourgeoisie juive assimilée et libérale de Vienne dont tous deux reçoivent l'éducation et la formation. Lorsque Schnitzler et Hofmannsthal émaillent leur propos de tournures en français ${ }^{9}$, c'est moins le signe d'une forme appuyée de snobisme que la trace de leur cosmopolitisme, de leur lecture des auteurs dans le texte original et de leur goût indéniable pour la langue française. Alors qu'il n'a que dix-sept ans, Hofmannsthal traduit en 1891 la pièce de Maeterlinck, Les Aveugles. Schnitzler ne s'aventurera pas dans ce type d'exercice mais prêtera à la traduction de ses écrits un intérêt soutenu, cherchant constamment à améliorer les traductions existantes de ses textes. Une fois qu'il aura choisi de délaisser la médecine pour persévérer dans la carrière littéraire, il utilisera ses contacts en France (P. Goldmann notamment) pour faire traduire ses textes. Henri Albert (Weihnachtseinkäufe / Emplettes de Noël), Jean Thorel (Liebelei/ Amourette), Maurice Vaucaire (Die Gefährtin/La Compagne) seront les premiers traducteurs à le faire connaître. Ses échanges avec Stephan Epstein, Maurice Vaucaire, Paul Géraldy plus tard, portent certes sur des questions de mise en scène mais, en tout premier lieu, sur la traduction des œuvres à faire connaître en France.

Dans la correspondance entre Schnitzler et Hofmannsthal, c'est en vain que l'on cherche la trace de discussions poussées ou approfondies sur la langue française ou la traduction ; des indices, dans les échanges avec S. Epstein notamment, permettent cependant de supposer que ces questions s'invitaient dans leurs conversations. Ainsi Schnitzler

9. - Ainsi Hofmannsthal trouve-t-il « vieux jeu », en français dans le texte, une nouvelle de Robert Ehrhardt, dans Hugo von Hofmannsthal Arthur Schnitzler, Briefwechsel, op. cit., p. 30. Dans la lettre du 1508 1930, Schnitzler écrit à Suzanne Clauser : „Gestern abends bei Julius en famille, der morgens aus St. M. ankam“ in Arthur Schnitzler, Briefe 1913-1931, op. cit., p. 702. 
soumet-il à S. Epstein la proposition à laquelle Hofmannsthal a songé pour la traduction du titre "Freiwild » et qui semble le satisfaire. Mais «Chasse libre » n'emporte guère l'adhésion de S. Epstein qui commente ainsi la suggestion: «klingt ja ganz nett ist aber absolut unfranzösisch $\gg 10$.

Il semble que ce soit en référence à des personnalités telles que $\mathrm{S}$. Epstein que Schnitzler se forge un avis sur la qualité des traductions et des traducteurs de ses textes. Lorsqu'il fait mention à Suzanne Clauser de ses réserves sur Maurice Rémon, Wilhelm Bauer et Alzir Hella 11 - le traducteur à qui Stefan Zweig réservait toute son estime - on peut supposer qu'il reprend à son compte l'avis d'un S. Epstein, ce dernier ne se privant pas de critiques rédhibitoires sur les traducteurs. Schnitzler ne prend pas forcément pour argent comptant les avis de ses intermédiaires mais en dernier ressort, et probablement aussi en raison de sa maîtrise malhabile du français ${ }^{12}$, il semble ne pas avoir d'autre choix que de faire confiance à ses interlocuteurs, à leur autorité et au crédit dont ils jouissent. Loin de négliger la question de la traduction, Schnitzler semble avoir pour priorité de porter son œuvre à la connaissance du public français. À cette fin, il est plus que probable que le critère de choix de la bonne traduction ait été celui qui a longtemps prévalu au $\mathrm{XIX}^{\mathrm{e}}$ siècle et perduré assez loin dans le $\mathrm{XX}^{\mathrm{e}}$ : un texte recevable pour un public étranger, un texte «domestiqué », passé sous la lentille de verres transparents, pour reprendre l'image de Georges Mounin.

Vu ainsi, la qualité de la traduction pourrait bien n'avoir pas été un but en soi mais un moyen pour se faire connaître du public français. Entraîné par le succès du Perroquet vert au Théâtre Antoine en 1903, Schnitzler se prend à rêver et demande à $\mathrm{S}$. Epstein si une programmation en province est envisageable ${ }^{13}$. En outre, sa correspondance le montre toujours soucieux de faire découvrir à l'étranger les différents registres de son œuvre. Schnitzler souffre en effet de n'être connu en France que comme auteur de Anatol ou de La Ronde et de s'y voir estam-

10. - Lettre du 15.07.1904. In Briefe von Stephan Epstein an A. Schnitzler, 19021908 Handschriftenabteilung Deutsches Literaturarchiv, Marbach. Après avoir songé à « Hallali » (cf. lettre qui pourrait être de juin 1904, cf. sources sus-nommées), Epstein retiendra finalement le titre « Le privilège ».

11. - Cf. Lettre à Suzanne Clauser du 15.08.1930, in Arthur Schnitzler, Briefe 1913-1931, S. Fischer, 1984, p. 702.

12. - Schnitzler maniait très bien le français mais des imperfections dont il était probablement conscient ne l'autorisaient pas à se dispenser du secours de traducteurs. À titre d'exemple, citons la lettre du 15.01.1923 à Paul Géraldy dans laquelle Schnitzler écrit : « Madame Zuckerkandl me raconte qu'on s'intéresse surtout pour La Ronde et moi je voudrais plutôt qu'on jouerait mes autres drames ». In cf. note précédente.

13. - In lettre du 19.11.1903 in Briefe von A. Schnitzler an Stephan Epstein, 19021908 Handschriftenabteilung Deutsches Literaturarchiv, Marbach. 
pillé auteur sulfureux et immoral. Dans une lettre à Berta Zuckerkandl du 4 mars 1929, il écrit qu'il serait pleinement satisfait si, avant de porter La Ronde à la scène, le théâtre Antoine pouvait montrer au public d'autres drames de son répertoire et faire évoluer ainsi sa réputation qu'il récuse ${ }^{14}$. Berta Zuckerkandl jouera de ses relations à Paris pour faire sortir du seul cercle des initiés le Schnitzler méconnu, le prosateur. Dans ce même effort, la rencontre avec sa traductrice Suzanne Clauser trois ans avant la disparition de Schnitzler, sera déterminante. Alors qu'il est au soir de sa vie et à l'apogée de son succès littéraire dans sa terre d'origine, S. Clauser ouvrira à Schnitzler la possibilité de conquérir une place en « terre étrangère » tout autant comme prosateur et nouvelliste que comme auteur dramatique.

La correspondance de Schnitzler reste le plus souvent elliptique sur les auteurs français ou les œuvres qui ont pu le marquer et se limite généralement à la citation de noms d'auteurs ou de titres d'œuvres. Il suffit cependant de songer au rôle clé de Hermann Bahr dans la diffusion à Vienne des auteurs français de la même période, pour comprendre que cette concision, loin de renvoyer à une connaissance lacunaire ou, encore moins, à un manque d'intérêt, indique bien plutôt que tous les auteurs évoqués sur le mode allusif participent de ce fonds culturel qui nourrit la génération de la Jeune Vienne, donne à sa réflexion esthétique son grain à moudre. Véritablement assoiffé de représentations théâtrales, Schnitzler fréquente assidûment les scènes de Vienne, Berlin ou Prague, ce qui lui vaut, tout comme sa liaison avec la comédienne Marie Glümer, de bien connaître le théâtre de boulevard. Si l'on ajoute bout à bout les noms cités par Schnitzler des auteurs de vaudevilles et de boulevard, la liste finit par être impressionnante : Alexandre Bisson, Victorien Sardou, Raoul Toché et Ernest Blum, Henri Meilhac, Edouard Pailleron, Paul Hervieu, Maurice Donnay, Abel Hermant, Gustave Guiche(s), Henry Bataille, Pierre Berton et Charles Simon, Henri Lavedan, Georges Courteline, pour s'en tenir à quelques exemples d'auteurs qui lui sont familiers. À ces noms s'ajoutent les grands classiques français que Schnitzler connaît bien. Comme nombre de ses contemporains de la Jeune Vienne, il apprécie Guy de Maupassant qu'il n'hésite pas à placer au même niveau qu'un Nietzsche ou un Goethe ${ }^{15}$. Parmi les autres prosateurs, il tient Anatole France en très grande estime. Cela dit, le caractère général et laconique de ces remarques laisse le lecteur quelque peu sur sa faim. Mais Schnitzler entre suffisamment rarement dans le détail d'une critique pour que l'on ne manque pas de relever

14. - Cf. Lettre à Berta Zuckerkandl du 04.03.1929, in Arthur Schnitzler, Briefe 1913-1931, op. cit., p. 594.

15. - Cf. Lettre du 21.06.1895, in Arthur Schnitzler, Briefe 1875-1912, op. cit., p. 262. 
certains traits frappants. Nous retiendrons deux aspects pour leur caractère récurrent : la sévérité du jugement émis et l'attention particulière portée à la question du style.

Alors que Schnitzler parle d'A. France en termes très élogieux, son jugement devient nettement plus réservé lorsqu'il évoque la pièce Musotte dont la représentation le déçoit beaucoup. La faute en incombe surtout à une mise en scène ratée et à une distribution catastrophique, écrit-il à Marie Glümer, mais il finit par concéder que la pièce n'est pas très bonne ${ }^{16}$. Globalement, les jugements réservés l'emportent sur les impressions enthousiastes et l'adhésion est le plus souvent tempérée d'un « oui mais ». Ainsi évoque-t-il dans une lettre à Richard Beer-Hofmann « diese hübschen Kleinigkeiten von Lavedan, von Courteline ${ }^{17}$. Lors de son séjour parisien en 1897, il déborde d'enthousiasme pour cette ville qui, selon son expression, « bouillonne de culture ». Mais s'il accepte de s'entendre dire par les Parisiens à propos de Vienne que «c'est une gentille petite ville », le jugement qu'il en retire sur le thêâtre parisien fait comprendre que l'humilité n'altère pas le tranchant de son esprit et qu'un recul particulièrement critique accompagne ses observations. S'il s'avoue séduit par le jeu des acteurs, il l'est beaucoup moins par l'art dramatique en général tel qu'il le voit pratiqué sur les scènes parisiennes. Voici ce qu'il écrit à Otto Brahm en 1897:

Von der hiesigen Schauspielkunst habe ich große Eindrücke bekommen, sehr mäßige von der dramatischen Kunst. Man spielt Feuilletons und Leitartikel -: aber diese Dinge versuchen kaum Stücke zu sein. [...] Ganz charakteristisch ist es, da $\beta$ beinahe alle Akte dieser Stücke in Zimmern spielen und da $\beta$ diese Zimmer den Blick [...] meist auf den eigenen Garten haben. ${ }^{18}$

Il est difficile de ne pas lire derrière cette critique une véritable profession de foi esthétique. En lieu et place de pièces de théâtre, dit-il, « on joue des feuilletons et des éditoriaux ». On entend que Schnitzler condamne un certain intellectualisme qui bannit de l'art dramatique la vie et la simple réalité au profit de la polémique ou du règlement de comptes. Il n'adhère pas à une forme d'art qui ne voit pas au-delà de son propre univers, de son propre jardin, pour reprendre son image, un art qui pèche, autrement dit, par nombrilisme ; et le lecteur d'aujourd'hui

16. - Lettre à Marie Glümer du 13.11.1892, in Arthur Schnitzler, Briefe 18751912, S. Fischer, 1981, p. 144.

17. - Cf. Lettre à Richard Beer-Hofmann du 26.04.1897, in Arthur Schnitzler, Briefe 1875-1912, op. cit., p. 317.

18. - Lettre à Otto Brahm du 23.05.1897, in Arthur Schnitzler, Briefe 1875-1912, op. cit., p. 323. 
se réjouit d'entendre Schnitzler dénoncer, entre autres, et probablement sans le savoir, le mal endémique du parisianisme.

Cette lettre en forme de compte rendu à $\mathrm{O}$. Brahm éclaire sur la volonté de Schnitzler d'affirmer son originalité, de frayer sa propre voie et de résister à toute influence ; le théâtre parisien lui offre un plaisir qu'il ne boude pas mais ses impressions pourraient se résumer à cette leçon : du goût pour la fantaisie française oui, un modèle certainement pas. Sur cette question de l'influence visiblement importante pour lui, il s'est déjà exprimé dans une lettre du 21 juin 1895 dans laquelle il parle de Nietzsche, admet l'impact inévitable de ce grand esprit sur la création moderne tout en rajoutant : « aber ich erkenne die Pflicht eines Dichters nicht an, sich von irgend einem großen Geist beeinflußen lassen zu müssen. ${ }^{19} \mathrm{Il}$ ne suffit certes pas de se défendre d'un travers pour garantir que l'on n'y succombe pas, mais cette volonté d'être soi-même par-delà toute influence résume assez bien l'ambition de Schnitzler, elle lui sert de ligne de conduite esthétique et morale. Aussi grand que soit un écrivain ou un penseur, il ne voit pas que le devoir d'un écrivain soit de se laisser influencer. D'où l'on conclut que le vrai devoir de l'artiste à ses yeux est d'être soi-même. Il est d'usage de louer chez le Schnitzler prosateur l'inventeur de la technique du monologue intérieur magistralement illustrée dans sa nouvelle de 1900, Leutnant Gustl. Un an après sa parution, Schnitzler écrit dans une lettre du 11 juin 1901 à son ami le philosophe et essayiste danois Georg Brandes : «Il existe une nouvelle de Dostoïevski, Krotkaïa (La Douce, 1876), que je ne connais pas et qui, semble-t-il, a recours à cette technique de pensées restituées sous forme de monologue. En ce qui me concerne, c'est une histoire de Dujardin intitulée les lauriers sont coupés qui m'a incité à user de cette forme. À la différence près que cet auteur n'a pas su trouver pour cette forme le bon contenu. » Voilà bien, tout en reconnaissant sa dette, une manière appuyée de revendiquer son originalité : « [Dujardin] n'a pas su trouver pour cette forme [le monologue intérieur] le bon contenu. » Le modèle peut être source d'inspiration mais chaque création doit être le fait de son créateur. On peut aisément imaginer ici que le «bon contenu » auquel Schnitzler se réfère dans la lettre à $\mathrm{G}$. Brandes réside dans ce que Hermann Bahr appelait en 1891 la « nouvelle psychologie » dans son essai Die Überwindung des Naturalismus, celle qui se recentre sur les états d'âme et les émois d'un sujet soudain devenu étranger à lui-même. Cette «nouvelle psychologie » qui a sa source chez un Paul Bourget, cherche à saisir, selon $\mathrm{H}$. Bahr, ce qui se passe au niveau des nerfs et des sens à l'état brut et originel, avant même l'appréhension de ces processus dans la conscience, sous la forme claire d'un sentiment. « $\mathrm{Si}$

19. - „Pflicht“" est souligné dans le texte in Lettre à N.N. du 21.06.1895, in Arthur Schnitzler, Briefe 1875-1912, op. cit., p. 262. 
donc », poursuit $\mathrm{H}$. Bahr, « la nouvelle psychologie veut s'épanouir à la première personne, les cas exceptionnels, ceux dont le dédoublement [est] maladif, [ceux] qui se demandent, sans cesse, comment ils sont émus et s'ils sont émus (Paul Bourget), doivent devenir sa règle. ${ }^{20}$

Le jugement un tantinet hautain de Schnitzler sur Dujardin fait par ailleurs ressortir tout le poids qu'il accorde à la forme. Si Dujardin n'a pas trouvé le bon contenu, un bon contenu ne serait rien sans la forme adéquate. Il y va ici du monologue intérieur mais la question de la forme se pose aussi par rapport à la langue. Et sur ce point, Schnitzler ne fait pas mystère de son engouement pour le français. A propos d'une lectrice enthousiaste dont lui parle sa traductrice Suzanne Clauser, Schnitzler recommande que cette admiratrice ne s'impatiente pas de le lire en allemand parce que ses nouvelles sont bien plus belles en français ${ }^{21}$. Schnitzler détestait se relire mais le faire dans une traduction française pouvait transformer une épreuve en plaisir. Voici ce qu'il écrit en 1903 à son ami Hofmannsthal à propos de la nouvelle Ehrentag parue sous le titre Jour de gloire: «Ces écrits rendent bien mieux dans une traduction française correcte que dans mon allemand $\gg 22$.

S'il est des langues, des styles et des formes qui servent mieux un contenu, c'est dire qu'ils sont là pour servir une intention et que chaque œuvre est mue par une finalité qui doit être décelable pour le lecteur et doit l'aider à l'éclairer sur les personnages, mais surtout sur lui-même. Dans la droite ligne de la philosophie des Lumières, Schnitzler apparaît, nous y reviendrons, comme l'héritier d'une littérature qui vise à instruire son lecteur, à lui renvoyer en miroir ses défauts, ses failles et à tenter de le remettre sur la voie d'une pacification avec lui-même. Nous verrons également plus avant que l'intérêt de Hofmannsthal pour la langue française ne s'aiguise pas exclusivement au vu des moyens esthétiques et stylistiques qu'elle offre mais plus largement au vu de sa fonction auprès des hommes qui la parlent.

20. - Cf. „Wenn also die neue Psychologie sich in der „Ich-Form“ erfüllen will, dann schränkt sie sich auf die Leute vom Metier ein“, qui tâtent le pouls à leur sensibilité, und die Ausnahmen mit dem kranken dédoublement, qui se demandent, sans cesse comment ils sont émus et s'ils sont émus (Paul Bourget), werden ihre Regel." Cité dans Evelyne Polt-Heinzl, Erläuterungen und Dokumente Arthur Schnitzler Leutnant Gustl, Stuttgart, Reclam, 2000, p. 33.

21. - Lettre à Suzanne Clauser du 15.08.1930, in Arthur Schnitzler, Briefe 19131931, op. cit., p. 702.

22. - „Diese Sachen - ich hab es neulich am „Jour de Gloire“ erfahren, wirken in anständiger französischer Übertragung besser als in meinem Deutsch. Die reine Tendenz des Erzählens ist dem romanischen Sprachgeist eingeboren, während er im deutschen gleichsam wie gegen die Natur wirkt, wenn die Mitteilung von Tatsachen der Seele und Menschlichkeit entbehrt", in Hugo von Hofmannsthal Arthur Schnitzler, Briefwechsel, op. cit., p. 179. 
Nous avons été frappé par la sévérité des jugements de Schnitzler, tant sur les autres que sur soi, frappé par sa propension à admirer, certes, mais du bout des lèvres. C'est que la critique est bien, pour Schnitzler, une question particulièrement sensible. Elle reste en effet étroitement liée aux doutes qu'il a entretenus sa vie durant sur ses propres qualités d'écrivain. Irene Lindgren commente ainsi les jugements de Schnitzler tirés de son Journal et qui ont trait à la création littéraire:

Wer in dieser Zitatensammlung blättert, kann nicht umhin zu merken, da $\beta$ die stärksten Triebfedern für Schnitzlers überkritische Einstellung zu sich selbst fehlendes Selbstvertrauen, Minderwertigkeitsgefühle und Unzufriedenheit mit der eigenen literarischen Leistung sind ${ }^{23}$.

Cette fragilité jouera indéniablement son rôle dans l'épisode particulièrement orageux de l'amitié entre Schnitzler et Homannsthal à propos de Der Weg ins Freie. Nous ne relèverons ici de cette brouille que la fureur froide avec laquelle Schnitzler va alors défendre son roman, son « enfant », comme il le dira, usant d'une image qui est tout sauf anodine. En effet, se défendre des attaques revient, vu ainsi, à défendre sa personne, à ne pas vouloir la dissocier de l'œuvre créée, c'est-à-dire, surtout, à se sentir responsable d'elle. S'il y a bel et bien entre l'œuvre et son auteur la différence qui sépare le père de l'enfant, il y a néanmoins aussi entre les deux un lien qui engage la responsabilité de l'auteur, voire de l'homme derrière l'auteur, c'est là une question morale que Schnitzler n'a jamais perdue de vue. Le besoin de compenser un manque d'assurance par l'attente d'une critique attentive et ouverte à toutes les subtilités de sa production littéraire revêt, au-delà de l'aspect psychologique, un aspect moral; il correspond à la volonté de répondre de son œuvre, tout en élaborant une œuvre qui appelle elle-même à l'éveil de la conscience, souvenons-nous du constat qui réconcilie Albertine et Fridolin à la fin de Traumnovelle : « Nun sind wir wohl erwacht », sagte Albertine, « für lange ».

C'est avec la même vigilance facilement ulcérée que Schnitzler surveille la critique de ses œuvres en France. La lettre-réquisitoire qu'il adresse à Edmond Jaloux après la parution dans les Nouvelles littéraires de la critique de Fräulein Else ${ }^{24}$ n'a d'égal, dans sa virulence, qu'un très fort besoin de reconnaissance. Ses commentaires sur la préface de

23. - Cf. Irene Lindgren, «Seh'n Sie, das Berühmtwerden ist doch nicht so leicht! » Arthur Schnitzler über sein literarisches Schaffen. Ausgewählt, kommentiert und mit einem Vor- und Nachwort von Irene Lindgren, Peter Lang, 2002, p. 18-19.

24. - Lettre du 24.10.1926, in Arthur Schnitzler, Briefe 1913-1931, op. cit., p. 463. A. Schnitzler reproche à E. Jaloux de n'avoir rien compris de l'intérêt de ce récit, de sa nouveauté. 
Félix Bertaux au recueil La Pénombre des âmes ${ }^{25}$ trahissent la même attente déçue.

Chez Hofmannsthal, le besoin de reconnaissance ne prend absolument pas les mêmes proportions car il n'est pas rongé par les mêmes doutes. La crise qui fait surface en 1902 au moment de la Lettre de Chandos se situe bien au-delà de la psychologie, elle ne relève pas d'une quête de soi mais d'une aspiration à l'adéquation parfaite entre l'art et les moyens qu'il met en œuvre, entre la recherche de la vérité et la vérité, la fiabilité, la justesse de ses formes d'expression. Et pour ce qui est de sa réception en France, Hofmannsthal va trouver, à la différence de Schnitzler, en la personne de Charles Du Bos, son passeur attitré qui dès 1927 fait entrer une partie de ses écrits dans La Pléiade ${ }^{26}$.

Toujours à la différence de Schnitzler, on peut penser que la France est source de retrouvailles avec soi pour Hofmannsthal. C'est en effet en 1900, au terme de son premier séjour à Paris, après avoir fréquenté hommes de lettres, artistes et théâtres qu'il rentre à Vienne affermi dans sa vocation : il n'embrassera pas la carrière universitaire mais se destinera à la littérature. Cette rupture n'a rien à voir avec le choix générateur de mauvaise conscience que représente pour Schnitzler l'abandon de sa carrière de médecin. L'engagement pour la littérature s'inscrit chez Hofmannsthal dans la continuité d'une quête, d'une recherche dont il décrypte une forme de parachèvement dans l'œuvre de Victor Hugo. La citation qui termine son essai de 1891 sur Maurice Barrès « Je suis perdu dans le vagabondage, ne sachant où retrouver l'unité de ma vie » 27 témoigne de sa préoccupation de ne pas céder aux sirènes du décadentisme et du dilettantisme et de retrouver l'unité du moi. Charles Du Bos avait fait, rappelons-le, l'éloge du « poète traversé ». Dans l'essai de 1906, «Der Dichter und diese Zeit », le poète apparaît tel un mendiant qui ne vit que de l'offrande des autres, il rassemble en lui ce qui est épars, il rend sa cohésion au présent éclaté. C'est là en partie une leçon que Hofmannsthal tire de sa fréquentation avec V. Hugo. Une autre rencontre est particulièrement fertile, c'est celle avec l'œuvre de Balzac. Hofmannsthal admire chez Balzac une œuvre qui, tout en faisant place à la profusion, à la masse imposante de personnages, de situations et de comportements, révèle une organisation, une force créatrice qui sait mettre en ordre et agencer tout ce matériau foisonnant :

25. - « Das Vorwort von Bertaux ist nicht sehr tiefsinnig. Und er wei $\beta$ überhaupt nichts davon, dass ich unter anderem auch Theaterstücke geschrieben habe ! », Lettre à Suzanne Clauser du 12.01.1930, in Arthur Schnitzler, Briefe 1913-1931, op. cit., p. 650.

26. - Cf. Hugo von Hofmannsthal, Écrits en prose, traduction de E. Hermann, La Pléiade - J. Schiffrin, Paris, 1927.

27. - In Hugo von Hofmannsthal, Reden und Aufsätze I 1891-1913, Gesammelte Werke, Frankfurt am Main, Fischer Taschenbuch Verlag 2166, erweiterte Ausgabe, 1979, p. 126. 
«Aus der Wahrheit der Myriaden einzelner Phänomene ergibt sich die Wahrheit der Verhältnisse zwischen ihnen : so ergibt sich eine Welt ». Et Hofmannsthal de souligner la cohésion de l'univers ainsi recréé : «Wie bei Goethe fühle ich mich hier in sicherem Bezug zum Gesamten ${ }^{28}$. La puissance d'un Balzac est titanesque parce que rien du temps présent n'échappe à son analyse mais son génie consiste encore à faire lever cette pâte qu'il modèle, ce qu'elle contient d'universel et d'universellement humain.

Cette aspiration à l'unité et à l'universalité, déjà tellement présente dans la poésie de Hofmannsthal ${ }^{29}$, se retrouve jusque dans ses impressions de voyage. Lors de son premier voyage dans le Midi de la France en 1892, c'est la beauté vivante des femmes de la Rome antique qu'il décèle derrière la silhouette des Arlésiennes. Un lien d'ombre, pour reprendre l'image de Jean-Yves Masson, relie bien le passé et le présent. L'ombre projetée des silhouettes mauresques révèle les beautés antiques ; derrière l'ombre du présent, brille, réconfortant, l'éclat du passé toujours vivant.

L'unité, c'est encore dans la langue française que Hofmannsthal la trouve préservée. Il admire les poètes de la Pléiade pour avoir su remodeler la langue française et être parvenu à créer, à partir de celle-ci, une tradition. Cette réflexion sur le rôle de la langue dans la construction et la cohésion d'une nation ne l'abandonne jamais. Dans l'essai de 1927, "Wert und Ehre deutscher Sprache », il regrette encore que fasse défaut chez les Allemands une "langue moyenne », selon son expression, celle-là même qu'il envie aux Français parce qu'elle relie, dans le même anneau l'écrivain et le lecteur, le poète et la nation ${ }^{30}$. La langue et, au-delà, les écrits dont elle est le matériau initial, servent de liant à ce grand organisme vivant et changeant que doit être une société pour constituer une nation. C'est dire la responsabilité de l'écrivain et le rôle qui lui incombe s'il ne veut pas se détacher de la société, se retirer dans une solitude peut-être splendide mais coupable car elle le mettrait en situation d'empêcher les membres d'une communauté de naître, de grandir et d'exister ensemble.

Euvrer à faire émerger la nation, à préserver le cœur vivant d'une communauté humaine, voilà bien la responsabilité qui revient à

28. - In „Balzac“, in Reden und Aufsätze I 1891-1913, op. cit., p. 390.

29. - „Und mein Teil ist mehr als dieses Lebens / Schlanke Flamme oder schmale Leier“ in „Manche freilich“ // „Und jedes Menschen wechselndes Gemüt, / Ein Strahl ists, der aus dieser Sonne bricht, / Ein Vers, der sich aus tausend andre flicht in „Was ist die Welt ?",, in Hugo von Hofmannsthal, Gedichte Dramen I 1891-1898, Gesammelte Werke, Frankfurt am Main Fischer Taschenbuch Verlag 2159, erweiterte Ausgabe, 1979, resp. p. $26 / /$, p. 86 .

30. - Cf. „Das Schrifttum als geistiger Raum deutscher Nation“, in Hugo von Hofmannsthal, Reden und Aufsätze III 1925-1929, Aufzeichnungen, op. cit., p. 26. 
l'écrivain allemand et à l'écrivain autrichien issu de l'Empire austrohongrois : face à l'éclatement de l'État multinational, Hofmannsthal persiste à nourrir le rêve d'une nation unifiée et réconciliée avec ellemême. Dans la conférence de Munich du 10 janvier 1926, il veut croire à l'unité d'une nation, à sa possibilité, dès lors qu'Allemands et Autrichiens sauront, à l'exemple des Français, se doter d'une littérature qui sera, à un niveau spirituel, le reflet, la traduction de leur réalité sociale et politique laquelle à son tour, ne sera réalité que si elle s'insère dans un espace spirituel ${ }^{31}$.

Ces considérations nous montrent l'abîme qui peut séparer un Hofmannsthal d'un Schnitzler quant aux raisons de leur intérêt profond pour la langue et la littérature françaises. Même s'il rêve lui aussi d'une Europe unie et pacifiée, Schnitzler est bien étranger à cette forme d'unité que Hofmannsthal appelle de ses vœux. Si son œuvre est celle d'un écrivain conscient de sa responsabilité dans le jeu de dupes et de cache-cache qu'une société sans autre repère que ses valeurs fissurées se joue avec elle-même, elle se situe en-deçà de toute mystique, elle ne vise pas une alchimie d'où une nation entière pourrait sortir régénérée et toujours plus grandie. On peut aller jusqu'à dire que son intérêt pour la France est celui d'un témoin de la modernité ouvert au souffle nouveau qui balaie alors la littérature européenne ; par contre, c'est dans la culture française, dans les institutions et les organes qui la représentent que Schnitzler aspire, semble-t-il, à trouver une place d'honneur et une large reconnaissance, celle qui pourrait éventuellement estomper ses propres doutes. Hofmannsthal trouve en Charles Du Bos son laudateur idéal, Schnitzler lui, restera longtemps trop parisien pour le public viennois mais hélas encore trop viennois pour le public parisien.

Mais poussons un peu plus avant cet éclairage parallèle du lien à la France de ces deux représentants magistraux de la modernité. À sa mort survenue brutalement, Hofmannsthal laisse derrière lui avec Andreas un roman de formation inachevé et, plus probablement encore, proprement inachevable. Alors que le moteur de toute son œuvre est une quête d'unité du moi avec le monde et avec soi-même, ce roman commencé en 1907 et constamment remis sur le métier consacre l'impossible unité du moi. Dans le roman resté fragment, c'est la fragmentation même qui devient matière du roman, le déborde, se dérobe à sa mise en forme ordonnée et policée, la rend impossible. Le terme du voyage initiatique qui mène le héros jusqu'à Venise est lui aussi repoussé toujours plus loin, ne révélant que masques ricanant sous d'autres masques,

31. - Cf. ce qu'il dit encore des Français : « Nichts ist im politischen Leben der Nation Wirklichkeit, das nicht in ihrer Literatur als Geist vorhanden wäre, nichts enthält diese lebensvolle, traumlose Literatur, das sich nicht im Leben der Nation verwirklichte.» In idem, p. 27. 
ne promettant qu'une dissolution toujours plus radicale du moi. Le lecteur voit se défaire sous ses yeux l'unité du moi, tout comme les mots abstraits, tels des champignons moisis, se décomposaient sous la langue de Chandos. Toute la matière vivante et foisonnante que Balzac parvenait à organiser dans un tout organique et dynamique laisse place ici, dans une forme romanesque radicalement moderne, au pur chaos qu'il s'agissait de dompter.

C'est un récit fort noir, Flucht in die Finsternis, qui occupe Schnitzler une vingtaine d'années durant et n'est publié que quelques mois avant sa mort. Schnitzler y reprend le thème de la folie autodestructrice et meurtrière de sa première nouvelle Sterben. Il analyse pas à pas, tout à la fois avec la distance froide de l'homme de sciences et l'humanité d'un Büchner, l'aliénation d'un sujet lentement dépossédé de luimême, qui s'abîme dans la folie. Commentant le sort de ce malheureux, son ami le Docteur Leinbach voit dans ce que la médecine et la justice vont qualifier de délire de persécution « eine Flucht ins System aus der friedlosen Vielfältigkeit der Einzelfälle ${ }^{32}$. Il voit dans l'explication rationnelle une fuite dans la rationalité rassurante qui s'illusionne probablement elle-même, préfère ignorer ses limites et décréter le vrai et le faux à partir d'elles.

Moderne, Schnitzler ne l'est pas seulement pour avoir sondé les âmes à des profondeurs psychanalytiques et révélé leur parfaite ambiguïté. Il l'est encore par son invite à critiquer, à partir de la raison, les limites de cette même raison. Ainsi nous apparaît-il comme l'héritier critique de l'Aufklärung et comme l'héritier moderne d'une tradition française qui s'enracine elle-même dans le legs des Lumières. Il invite à inspecter sans complaisance la belle apparence de la réalité, tout autant qu'à analyser les outils qui servent à l'appréhender. Radicalement moderne, probablement contre son gré, Hofmannsthal l'est aussi, lui qui tout en cherchant à rester dans la filiation des grands récits qui construisent l'homme et le monde, déroule dans Andreas l'odyssée sans retour d'un moi éclaté en mille facettes et d'un monde en perdition.

Dans leur relation à la France, sa langue et sa littérature, Schnitzler et Hofmannsthal illustrent deux modes d'être fort opposés. Au rejet quasi viscéral de toute ressemblance tel qu'il s'affirme dans l'originalité que Schnitzler revendique, répond la filiation parfaitement assumée d'un Hofmannsthal. En atteste la première de Die grüne Flöte au Deutsches Theater de Berlin le 26 avril 1916. Avant le ballet, on porte à la scène ce soir-là la comédie en un acte Die Lästigen. Des critiques s'abusent et en attribuent la paternité à Molière sans soupçonner le moins du monde l'adaptation très libre des Fâcheux par Hofmannsthal. Celui-ci est ravi

32. - Cf. Flucht in die Finsternis, in Arthur Schnitzler, Erzählungen, Zürich, Manesse Verlag, p. 627. 
de voir sa véritable identité dissimulée, le temps d'un malentendu, sous le nom de Molière ${ }^{33}$. Il n'en reste pas moins que c'est à travers la divergence de ces modalités que chacun explore deux pointes extrêmes de la modernité : pour l'une, une rationalité mise en échec par une réalité que seules des frontières artificielles contiennent, pour l'autre une plongée en spirale dans l'abîme du temps et de l'espace mais d'où l'esprit pourtant « ébloui, haletant, stupide, épouvanté » ${ }^{34}$, comme chez Hugo, ne revient plus, à l'inverse de chez Hugo. C'est toutefois à partir de ces deux extrêmes que les deux se rejoignent, consultant en eux, comme le dit P. Valéry, « quelque chose d'étrangement actuel » et figurant au plus haut dans le Panthéon de la modernité, si ce n'est pas là abuser d'un oxymore.

33. - Cf. Elsbeth Dangel-Pelloquin «'Das kleine Falsificat'. Das Spiel von Original und Fälschung in Hofmannsthals Die Lästigen. Nach dem Molière ». In Hugo von Hofmannsthal-Gesellschaft Hofmannsthal-Jahrbuch zur europäischen Moderne Bd 10/2002, consultable en version pdf. (E. Dangel.pdf) à partir du site: http://www.arthurschnitzler.at/texte-und-bibliographie.html.

34. - Cf. le poème «La pente de la rêverie » in Victor Hugo, Euvres poétiques, Pierre Albouy (éd.), La Pléiade, t. 1, Paris, Gallimard, 1964, p. 774. 
